\title{
Research on Brand Remodeling and Marketing Strategy Optimization of Chinese Domestic Cosmetics in Post-pandemic Era
}

\author{
Lening Dong ${ }^{1, \dagger}$, Hanfei $\mathrm{Liu}^{2, \dagger}, \mathrm{Yi} \mathrm{Tao}^{3, *}, \dagger$ \\ ${ }^{1}$ Guangdong University of Foreign Studies, Guangdong, P.R.China. \\ ${ }^{2}$ Changzhou No.1 High School, Changzhou, Jiangsu, P.R.China. \\ ${ }^{3}$ Beijing Normal University- Hong Kong Baptist University United International College, Guangdong, P.R.China. \\ ${ }^{*}$ Corresponding author. Email: n830031172@mail.uic.edu.cn \\ These authors contributed equally.
}

\begin{abstract}
The Covid-19 pandemic has dramatically attacked the Chinese cosmetics industry, impeding its development by reducing consumption, which is an urgent problem that needs to be addressed as cosmetics have already become daily necessities for many people. Such a lag phase has negatively influenced many people as they stop work and earn less money than before, changing their consumption concept from luxuries to goods at lower prices, such as domestic cosmetics products. Most importantly, with the rise of social media, Chinese cosmetics brands must develop further in this field. This paper will respectively take advantage of case study, comparative study, and literature review analysis to analyze our topic. By these means, we find out two potential problems that current Chinese cosmetics brands have: unstable market influence and the lack of professional and technical personnel. To solve these problems, we develop some solutions for brands: focusing on R\&D and improving brand innovation awareness; seizing the market demand and creating brand differentiation points; endowing domestic cosmetics with cultural connotation, and expanding brand awareness. We hope this paper will offer Chinese cosmetics brands some theoretical and practical ideas to improve their marketing strategies during the post-pandemic era and help them successfully get through the COVID19 crisis. Also, we expect that our research will enlighten more people to explore ways of brand remodeling, regarding targeted audiences as a standard for quantifying results.
\end{abstract}

Keywords: Post-pandemic era, Chinese cosmetics, Brand remodeling, Marketing strategy

\section{INTRODUCTION}

The post-pandemic era is an era in which the COVID-19 pandemic will rise and fall from time to time, and small-scale outbreaks may occur at any time. It is not the situation that we originally imagined that the pandemic would disappear completely and everything would recover as before. It has had a farreaching impact on all aspects of human lives [1]. In the post-pandemic era, for the Chinese cosmetics market and industry, cosmetics consumption is still optimistic. With the popularity of the beauty economy, cosmetics have become an essential category for the younger generation. Although the shutdown and wage reduction during the pandemic affected the income of consumers, after the removal of masks, women's demand for beauty reappeared, and consumers' demand for cosmetics would rebound [2]. Besides, the online marketing mode and consumption, which were popular due to the advantages of non-contact and security during the pandemic period, will continue to be beneficial to brands in the post-pandemic era [3]. That is because generation $\mathrm{Y}$ and $\mathrm{Z}$, born from the 1980s to 1990s and from the mid-1990s to the early 2000s respectively [4] and are getting used to the convenient and efficient online new retail mode [2], are the main consumers' groups of cosmetics. Therefore, more and more domestic cosmetics brands will embrace the Internet, actively using short video implantation, online livestreaming, and other online ways to strengthen brand promotion to increase sales.

However, with the deepening of economic globalization and the expansion of the Chinese 
cosmetics market, Chinese cosmetics brands need to face competition from domestic brands and more and more foreign ones. According to the Beauty and Skincare Report of Little Red Book generated by ZDigital Technology Institution, in the Chinese cosmetics market, international brands are generally distributed in the "popular", "middle and high-end", "high-end" and "luxury" categories. In contrast, the vast majority of domestic brands are distributed in the two gradients of "middle and high-end" and "popular" [5]. This means that different from consumers' impression of the "highend atmosphere" of international cosmetics, consumers still have the stereotype of "low-end" or "outdated" of domestic cosmetics brands. Although the growth of domestic brands is driven by new content marketing online, with the international brands increasing the cost of marketing campaigns to increase their strong brand potential, the general marketing cost is bound to rise. It means it may be hard for domestic ones to keep on the same marketing strategy [6]. Therefore, for Chinese cosmetics brands, brand remodeling or reshaping would be an important proposition.

At present, the research on the post-pandemic era and Chinese cosmetics brands mainly focus on the following aspects.

In recent years, some domestic cosmetics are gradually rising, and they have been reshaping their brands with the help of new media. Qin and Xiu took Pechoin, an old Chinese cosmetics brand, as an example to analyze its brand remodeling and shortcomings in the economic, competitive, technological, social and cultural environment and its brand image in the new media era [7]. Taking CHCEDO as an example, Kan and Yuan summarized its attempts and shortcomings in brand innovation, variety broadening, popularity improvement, and brand combination [8]. Although some Chinese cosmetics are rising, there are still great difficulties. $\mathrm{Li}$ pointed out that domestic cosmetics tended to rise and disappear rapidly, while foreign brands had a longer influence on consumers. Due to the lack of long-term investment and time accumulation, it is difficult to compete with international brands in brand value [3].

In the post-pandemic era, great changes have taken place in the Chinese cosmetics market, bringing new development opportunities for domestic cosmetics. Gong said that after the pandemic, the consumption of cosmetics was relatively optimistic. When people no longer wear masks, women's demand for beautiful make-up will reappear. Especially with the influence of the live-streaming trend, the makeup market is prone to retaliatory consumption. But at the same time, due to the impact of the pandemic on the income of Chinese consumers, although consumers' demand for make-up will rebound, the unit price of customers will drop in a short period. It also makes consumers shift from buying high-end foreign brand makeup to more cost-effective domestic makeup [2]. Guo pointed out that after the pandemic, high-end cosmetics brands in Japan, South Korea, and other countries could not cope with the Chinese market, and there was a blank window period for middle and high-end cosmetics consumers. At the same time, the pandemic makes the patriotic feelings of the whole Chinese people rise, and national brands are favored by consumers [9]. Su said that due to the impact of the pandemic, many cosmetics brands have changed from offline sales to online sales, and the livestreaming commerce mode has developed rapidly during this period. At the same time, affected by the pandemic situation, the phenomenon of hierarchical consumption of cosmetics is more obvious. And he put forward two suggestions that cosmetics should pay more attention to the integration of online and offline channel marketing and pay attention to the segmentation of niche markets [10].

Based on the above research on the dilemma of domestic cosmetics and the development opportunity in the post-pandemic era, we find that few scholars are studying how to reshape the brand of domestic cosmetics in the post-pandemic era, compete with international brands, and put forward some corresponding marketing strategies according to the characteristics of cosmetics consumption in the postpandemic era.

\section{METHOD}

\subsection{Case Study}

The case study is a scientific method that helps researchers find out a profound and comprehensive understanding of complicated problems they want to solve and is widely used in various disciplines, especially in the social science field. The central tenet explores a phenomenon and its natural context, being a naturalistic design rather than an experimental design whose variables are controlled by investigators [11]. We use Perfect Diary and Pechoin as domestic cases, representing new and old brand categories in the modern Chinese cosmetics market. The choosing reasons are as follows.

Firstly, Perfect Diary is a new cosmetics brand that rises in China since 2017. Its young image provides fast fashion makeup products and beauty solutions for the new girls' generation with infinite energy, exhibiting domestic beauty brands' promising future. Characteristics that Perfect Diary has are common among a great number of young cosmetics brands. Therefore, Perfect Diary is a typical case for us to explore.

Secondly, Pechoin, one of the most established cosmetics brands set up in 1931 in China, is well-known 
for its functions of maintaining beauty and keeping young. We choose it as the case because it is representative, creating a brand image with characteristics of oriental Chinese tradition which can generalize to the old brand category.

Whether Perfect Dairy or Pechoin, they have done a great job by perfectly taking advantage of online ecommerce to increase sales volume. Nevertheless, in the long run, profits gained by this strategy brands will become less obvious. As a result, they need to create new marketing plans for their future by re-branding.

\subsection{Comparative Analysis}

A comparative analysis compares two or more subjects or ideas, showing how they are similar to or different from each other and creating debates. In this way, we can research objects or events from multiple perspectives and draw an all-sided conclusion [12]. In this paper, the horizontal comparison is applied. L'Oréal Paris, a famous French cosmetics brand, is chosen as the comparison object for Perfect Dairy and Pechoin. It is a strong competitor of Chinese domestic brands in market share and brand image.

\subsection{Literature Review Analysis}

We collected and re-explained relevant data and literal resources through the data reports released by CNKI, university library resources, and data platform. In this way, can we get a profound definition of brand remodeling from the perspective of the theory of communication, using study methods correctly and get a deeper understanding of what Pechoin, Perfect Diary, L'Oréal, and other cosmetics brands have done, analyzing their current status, past experiences, and future plans. Also, we can come up with new approaches and better state our views about Chinese cosmetics rebranding during the post-pandemic era [13].

\section{RESULT}

\subsection{Analysis of the Development Status of Chinese Cosmetics Brands in the Post- pandemic Era}

Under the impact of the pandemic, the Chinese cosmetics industry was violently attacked. From January to February in 2020, the sales volume of domestic cosmetics dropped about 20 percent compared with a year earlier. Not until online marketing campaigns appeared in March, customers' desires have been partially released, promoting a trivial Chinese cosmetics consumption. However, the transaction volume of the ecommerce platform revealed that the domestic cosmetics industry showed compensatory consumption rather than retaliatory consumption that experts had expected. Still, by the following several months, the sales volume did not take a turn for the better. [14] Till 2021, although sales volumes have increased in a small amount, there is still a gap between current volumes and volumes before the pandemic. Chinese cosmetics industry has been in a dilemma.

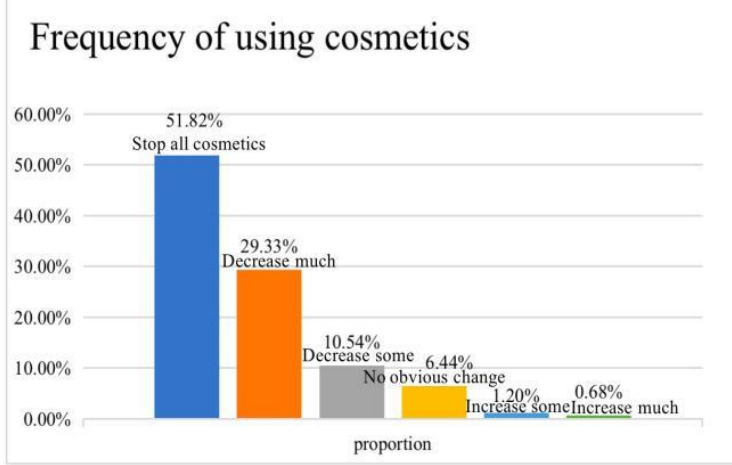

Figure 1 Frequency of customers using cosmetics during the pandemic era

\subsubsection{Challenges of brands}

Coronavirus greatly impedes customers' desires to purchase cosmetics. For the following long time, people need to spend most of their time staying indoors or going outside with thick masks to hide most of their faces. As a result, given in Figure 2, how to promote the selling of makeup products rather than skincare products will be a tricky problem for cosmetics marketers to solve.

Also, high-level cosmetics brands seize the opportunity and try to carve up Chinese cosmetics industry market shares simultaneously, making domestic brands have to enhance their competitiveness and become dominant.

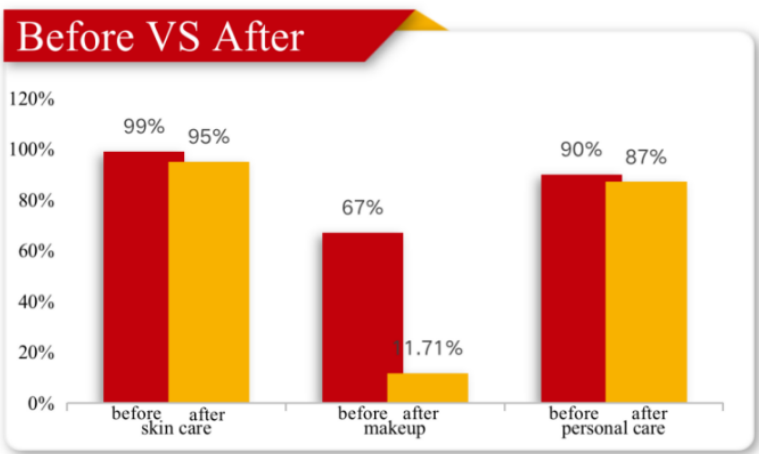

Figure 2 Comparison of the use proportion of different products before and after the pandemic 


\subsubsection{Chances of brands}

Firstly, because the COVID-19 pandemic decrease customers' purchasing power, Chinese people prefer domestic brands more than before because of their price performances and sophisticated marketing strategies.

At the same time, the pandemic tremendously accelerates the development of e-commerce and livestreaming, helping people do shopping online at any time they want to, which also offers an effective platform for cosmetics brands to increase their sales volume by cooperating with different kinds of social media platforms. Compared with traditional ecommerce, live streaming has stronger interaction between brands and customers, enhancing the display of products themselves and increasing the conversion rate [14].

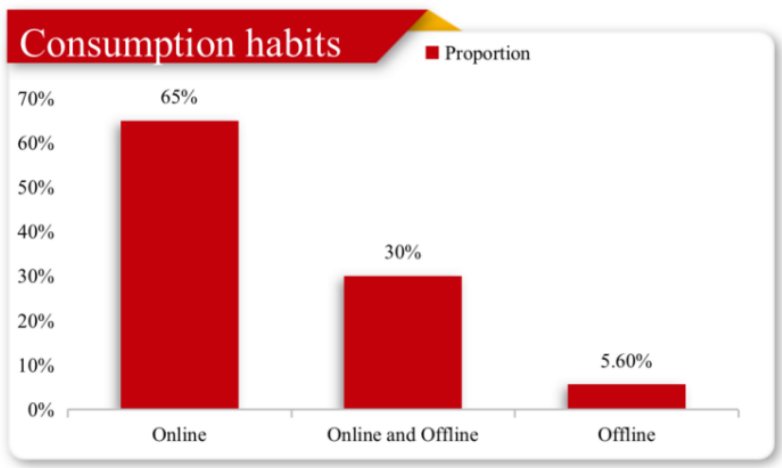

Figure 3 Daily purchasing channels customers use for cosmetics

Moreover, Chinese cosmetics brands can take the chance to simultaneously develop offline cosmetics marketing, building differentiation with others. Being in the first place to create new products or selling approaches is the best way to attract customers in a new turn. For instance, at the end of 2019, a makeup collection store called The Colorist firstly flourished in Shanghai, gathering various cosmetics brands and featuring "large-scale collection + fast fashion speed," [14] which was a great success during the postpandemic era.

\subsubsection{Customer analysis}

With the appearance of e-commerce and livestreaming, according to Figure 4, the cosmetics market attracts a great number of people who are at the age of 15 to 34 , being the main purchasing forces. In common, they regard cosmetics as necessities, having a middle and upper-level income.

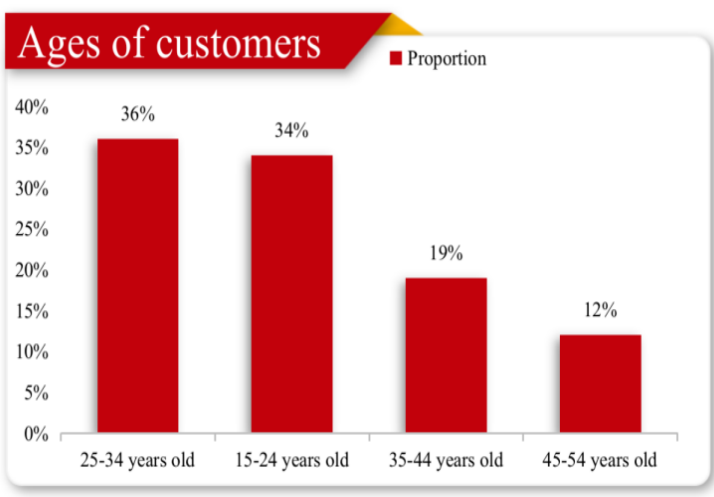

Figure 4 The Ages distribution of customers

\subsection{Brand Remolding Analysis of Chinese Domestic Cosmetics}

\subsubsection{Connotation and features of brand remolding}

Brand is the sum of the public's cognition of the enterprises and their products [15]. Brand communication is the communication behavior of brand owners who continuously and consistently convey brand information to the target consumers through advertising, marketing, and other means of communication, and hope to ultimately achieve the effect of meeting consumer demands and improving brand voice and assets. From the perspective of communication studies, the research scope of brand communication mainly includes "sender", "receiver", "message", "channel," and "effect" [16]. Since in the scope of our discussion, the "sender" and "receiver" are determined, and the "effect" cannot be controlled, brand remolding would mainly act on message and channel. "Message" refers to the content of brand communication, including brand culture, identity, and core value; "Channel" basically includes mass media and digital media [17]. Next, we will analyze and compare the brand shaping with the brand cases of Pechoin, Perfect Diary, and L'Oreal, focusing on the aspects of "message" and "channel".

\subsubsection{Analysis of brands in the modern Chinese cosmetics market}

\subsubsection{Perfect Diary}

Founded in 2017, Perfect Diary had successfully ranked among the first-line domestic brands with a market value of more than five billion dollars in only one and a half years. Its comprehensive index and ranking are also on the rise [18]. Its brand concept is unlimited beauty, and its brand vision is to advocate that "the younger generation should not be bound by external labels, but strive to break through themselves and meet a better self" [19]. As a brand that has only been established for three years, Perfect Diary's brand 
culture and connotation seem to lack competitiveness. However, its success lies in its accurate target positioning and comprehensive brand communication channel strategy [18]. Perfect Diary accurately locates its target audience in generations $\mathrm{Y}$ and $\mathrm{Z}$ who pursue personality, convenience, and experience. Compared with the consumption mode of pursuing luxury brand logos, they tend to choose products with more personality[20]. Perfect Diary captured the characteristics of this consumer group, so based on these characteristics, the communication strategy relying on the accumulation of social platform and private domain traffic was implemented. Key Opinion Leader (KOL) resources on Chinese social media platforms (TikTok, Little Red Book, Bilibili, and so on) have been regarded as the company's core competitiveness. Also, it has introduced public traffics into the private domain traffic pool based on WeChat social network system, maintaining the WeChat community through cultivating Key Opinion Consumers, launching low seckill activities, and so on to improve the repurchase rate of old customers and extend products' life cycle [18]. The effect of such a strategy was that it had reached about 40 million consumers, with a coverage rate of about $35 \%$ 40\% till March 2021 [21].

\subsubsection{Pechoin}

Pechoin is the oldest skincare brand in China, with the brand concept of "natural herbs without stimulation" [16]. Compared with the new domestic brands such as Perfect Diary, its biggest advantage lies in the national popularity and profound brand culture and connotation accumulated over the years, which means that the old domestic brands are more likely to arouse consumers' identification and emotional dependence on their own national culture [22]. Although in the minds of some consumers, Pechoin is out-of-date, it also uses digital communication means such as social media and ecommerce platforms to meet the consumption needs of the young consumer while guarding the brand classics. For example, in 2019, it launched products packaged with Dunhuang murals by co-branding with Dunhuang Museum. Such a tactic has improved brand awareness, maintain its own brand cultural advantages, and carry out brand rejuvenation transformation [22].

\subsubsection{Comparative analysis on the construction of domestic and international brands in the post- pandemic era}

The new domestic products represented by Perfect Diary rely too much on marketing to obtain traffic and exposure. According to the financial report of the third quarter of 2020, the marketing expenses of perfect diary reached 2.034 billion yuan, accounting for $62.16 \%$ of the total revenue. However, L'Oreal, with a market value of 204.9 billion US dollars, has never let its marketing expenses account for more than $15 \%$ in the past five years. Even in 2019, when the peak appears, it was only $20.3 \%$ [20]. At the same time, when the strategy of using new content marketing to drive growth becomes the basic operation, international brands will also spend a lot of money to promote. At this time, the marketing cost rises, and the new domestic brands must compete with international brands in brand value.

For the old domestic brands represented by Pechoin, although they have a long history compared with international brands, their brand image has not yet been transformed. Different from consumers' impression of the "high-end atmosphere" of international cosmetics, consumers still have the stereotype of "low-end" and "outdated" of domestic time-honored cosmetics brands.

\subsubsection{Problems in brand building of Chinese domestic cosmetics}

\subsubsection{Brand's market influence is unstable}

The brand potential energy of a brand refers to the market share of the product, the popularity and favor in consumption, and the relevance with the product category. Taking Perfect Diary as an example, domestic cosmetics have been at the forefront of Chinese cosmetic brands in recent years. The next step is inevitably to compete with international brands. But at present, the traditional international brands still occupy a wide market share. For example, L'Oreal Group owns Paris L'Oreal, Lancome, Maybelline, and other wellknown cosmetics brands. The latest financial report of L'Oreal shows that the annual sales in the Asia Pacific region in the first half of the year was 35.467 billion yuan, a year-on-year increase of $24.3 \%$. It can be seen that international cosmetics brands have high potential and still have great influence in the post-pandemic era. Although consumers may be reluctant to buy big brand cosmetics for economic reasons, young consumers will still choose international brands when they have more wealth with age. Domestic brands are still weaker than international brands with a long history in brand value.

\subsubsection{Lack of research and development ability $\underline{(R \& D \text { ability) }}$}

At present, most domestic cosmetics brands rely on original equipment manufacturer (OEM) manufacturers for formula supply. OEM manufacturers have a variety of formulas for each type of product. They 'develop' products according to the requirements of customers to choose the formula. An OEM manufacturer usually serves multiple brands in China and has no energy and time to develop products for specific brands. Therefore, the common selling point of many popular domestic cosmetics is that they are cost-effective or have an outstanding effect in one aspect. Thus, there is a gap 
between them and international brands in the overall sense of use.

There is a lack of $R \& D$ professionals in China's cosmetics industry. Many well-known domestic cosmetics brands will pay a lot of money to hire foreign R\&D personnel such as Japan and South Korea. Their own R\&D personnel is relatively lacking advanced concepts and experience.

\section{DISCUSSION}

We put forward the following points to reshape the domestic cosmetics brand optimization marketing strategy.

\subsection{Focus on $R \& D$ and improve brand innovation awareness}

In the long run, if cosmetic brands want to occupy a stable position in the market, they must have core competitiveness centered on technology or ingredients. At present, China's domestic cosmetics brands have spent a lot of effort in marketing, and their ability to grasp new channels is better than that of international brands. Next, a part of the marketing cost can be invested in product $\mathrm{R} \& \mathrm{D}$ and innovation, and the core technology and marketing means can be combined to improve the R \& D strength and R \& D system.

Clinique, an American cosmetics brand, is the first one to classify users' skin into different types, launch personalized consultation services, recommend products of corresponding skin types for users, thus winning the favor of consumers. Domestic brands should also consciously develop and design different products according to different people and skin types. The product quality and service quality of intensive cultivation make the product quality worthy of publicity and marketing advertising words.

\subsection{Seize the market demand and create brand differentiation selling points}

If domestic brands want to break through the current cosmetics market, they should pay attention to building their own differentiated selling points. This requires that the brand always follow up the needs of consumers, speed up the pace of product change, and evolve its own development model.

After the pandemic, people's awareness of health protection will be stronger than before, and the wearing of masks will still become the norm for a long time. Therefore, the research and development of products should also keep the properties of makeup and meet consumers' needs to wear masks. For example, the demand for eye makeup will continue to increase, and consumers will favor the rich, varied and personalized eye makeup. The durability and waterproof properties of eye makeup will also be a concern for consumers. In addition, long-term wearing masks will also make a face short of water and smallpox. Therefore, anti-allergy, acne suppression, and skincare liquid foundation will also greatly meet consumers' demand for cosmetic functions.

\subsection{Endow domestic cosmetics with cultural connotations and expand brand awareness}

In recent years, the Chinese fad has been loved by many young people. Commodities with Chinese cultural flavor can move Chinese consumers and arouse their sense of national pride and identity. For example, the Chinese style dressing box jointly named by Pechoin and the Forbidden City and the combination of Herborist and the concept of traditional Chinese medicine have won the love and attention of Chinese consumers.

Using this experience for reference, the development of domestic cosmetics brands can be based on Chinese traditional culture, integrate their own brand image with Chinese traditional culture, and distinguish them from foreign cosmetics. According to the characteristics of their own products, brands can co-name with traditional Chinese cultural products to create products with Chinese quality, which awakens the democratic spirit of consumers and gives people a refreshing feeling. In this way, we could carry forward Chinese traditional culture and take this opportunity to tell our own brand story and give the brand a more profound connotation.

\section{CONCLUSION}

In this study, we take Chinese domestic cosmetics brands - Perfect Diary and Pechoin as the research cases to analyze the existing marketing strategies they adopt to reshape their brand images and compare their brand images with L'Oréal. To sum up, the growth point of Chinese domestic cosmetics brands in the postpandemic era should focus on the domestic market competition, seize the characteristics of young consumers' pursuit of personality, constantly promote the upgrading of technology and marketing, and compete with international brands in the way of differentiated competition, to realize the leap of brands from low-end to high-end. Among them, quality and innovation are the first places, and then enterprises should also pay attention to maintaining Chinese cultural characteristics. It is hoped that domestic cosmetics enterprises could explore a unique selfdevelopment path in the post-pandemic era through deep insight into consumers, market, and technology trends.

In addition, there are also a few deficiencies in this paper. For example, in the problem analysis, we mainly 
use the method of case analysis and comparison, focusing on the analysis of brand marketing effect from the perspective of consumers, which makes the research dimension limited. In the future, we can increase interviews with industry experts and brands in the cosmetics industry to enrich research perspectives. Besides, combined with the relevant theoretical knowledge and cases, the brand communication strategy can be studied more comprehensively and deeply. More practical development suggestions may be put forward for more domestic cosmetics brands.

\section{REFERENCES}

[1] Wang Zhuli. How should education transform in the post-pandemic era[ J] Research on audio visual education, 2020041 (004): 13-20

[2] Gong Shuhui. The trend of China's cosmetics market in the post-pandemic era [J]. China cosmetics, 2020 (7): 20-24

[3] Wang Meng. (2020). How to seize the new development opportunity of cosmetics consumer market in the post-pandemic period. China cosmetics, No. 425 (12), 84-87

[4] Wood, S. (2013). Generation Z as consumers: trends and innovation. Institute for Emerging Issues: NC State University, 119(9), 1-3.

[5] Beauty and Skincare Report of Little Red Book. ZDigital Technology.

[6] Qin Ruiyu, Xiu Mei. The new media marketing strategy of China's time-honored cosmetics brands: a case study of Bai Queling [J]. News outpost, $2020(7)$

[7] Kan Bingbing, Yuan Limin. Research on marketing strategy of domestic cosmetics brand under new media environment -- taking natural hall as an example [J]. Western leather, 2021,43 (01): 131132

[8] Li Weilong. The perfect diary of new domestic cosmetics, the problems of huaxizi [J]. Sales and marketing (Management Edition), 2020, no.677 (03): 49-51

[9] Guo Li. Trend analysis of cosmetics market under pandemic situation [J]. China cosmetics, 2020, No. 417 (04): 86-91

[10] Su shuoran. Live delivery of cosmetics will develop towards standardization and specialization [J]. China cosmetics, 2020, No. 425 (12): 82-83

[11] The case study approach Sarah Crowe,corresponding author Kathrin Cresswell, Ann Robertson, Guro Huby, Anthony Avery, and Aziz Sheikh Published online 2011 Jun 27.
[12] What is Comparative Study? Syed Aftab Hassan Bukhari Posted: 21 Nov 2011 Last revised: 4 Dec 2011

[13] The Research on the Pechoin's Communication Strategy from the Perspective of 4I Theory, Yin peiyi, June, 2020

[14] Shuhui Gong. Post-pandemic Era Chinese Cosmetics Market Trends. China cosmetics review, 2020, 20-24

[15] Yu Mingyang, Zhu Jida, Xiao Junsong. Brand communication $[\mathrm{M}]$. Shanghai Jiaotong University Press, 2015.

[16] Yin Peiyi. Research on the brand communication strategy of Pechoin from the perspective of $4 \mathrm{I}$ theory [D]. Henan University, 2020.

[17] Duan Chunlin. Integrated brand communication: theoretical construction from IMC to IBC [M]. Guangzhou: World Book Publishing Guangdong Co., Ltd., June 2014.

[18] Wen Zhe. Marketing strategies of domestic cosmetics brands under New Media Marketing -Taking perfect diary as an example [J]. Office automation, 2021,26 (10): 30-32.

[19] Perfect Dairy official website. http://www.perfectdiary.com/index.php?c=categor y\&id=28

[20] Ye Yang, Wang Yue. Beauty brand marketing strategy in the Internet Environment: Taking perfect diary as an example [J]. Quality and market, 2020, \{4\} (19): 70-72

[21] The dilemma of Perfect Diary https://baijiahao.baidu.com/s?id=16939195049392 $29519 \& w f r=$ spider $\&$ for $=p c$

[22] Qin Ruiyu, Xiu Mei. The new media marketing strategy of China's time-honored cosmetics brands: a case study of Bai Queling [J]. News outpost, 2020, \{4\} (07): 107-109 